\title{
Dynamic Nuclear Polarization by Thermal Mixing Under Partial Saturation
}

\author{
Sami Jannin · Arnaud Comment • J. J. van der Klink
}

Received: 23 March 2012/Revised: 24 April 2012/Published online: 2 June 2012

(C) Springer-Verlag 2012

\begin{abstract}
We describe a low-temperature thermodynamic model for dynamic nuclear polarization (DNP) via continuous-wave partial saturation of electron spin resonance (ESR) lines that are both homogeneously and inhomogeneously broadened. It is a variant of a reasoning proposed by Borghini, which in turn used Redfield's thermodynamic treatment of saturation. Our variant is furthermore based on Provotorov's insight that under partial saturation of a coupled-spin system two distinct spin temperatures should appear in a thermodynamical theory. We apply our model to DNP results obtained at a temperature of $1.2 \mathrm{~K}$ and in magnetic fields of 3.35 and $5 \mathrm{~T}$ on $1-{ }^{13} \mathrm{C}$ labeled sodium acetate dissolved in a frozen $\mathrm{D}_{2} \mathrm{O} /$ ethanol- $\mathrm{d}_{6}$ solution doped with the free radical TEMPO.
\end{abstract}

Dynamic Nuclear Polarization (DNP) by thermal mixing can be described in thermodynamic terms $[1,2]$. The basic idea is that the electron spin Hamiltonian may consist of two commuting parts (typically the Zeeman and the truncated dipolar Hamiltonians) that cannot exchange energy, because the relevant quanta are not commensurate. However, when a suitable microwave field is added, the thermodynamics can be applied in a rotating frame, where the total Hamiltonian is timeindependent [3]. In that frame, the Zeeman and dipolar Hamiltonians do have commensurate quanta, and it is found that energy will flow out of the dipolar energy reservoir into the Zeeman reservoir, and from there, by spin-lattice relaxation, to the lattice. A stationary state may be created, where the dipolar reservoir has a

\footnotetext{
S. Jannin $(\bowtie)$

Institut des Sciences et Ingénierie Chimiques, Ecole Polytechnique

Fédérale de Lausanne (EPFL), Batochime, 1015 Lausanne, Switzerland

e-mail: sami.jannin@gmail.com
}

A. Comment · J. J. van der Klink

Institute of Physics of Biological Systems, Ecole Polytechnique

Fédérale de Lausanne (EPFL), 1015 Lausanne, Switzerland 
temperature considerably lower than that of the lattice. When furthermore the nuclear Zeeman Hamiltonian is considered it is again found that it may have commensurate quanta with the electronic dipolar reservoir: by nuclear-electron spin dipolar coupling the nuclear Zeeman reservoir is cooled as well and the nuclear polarization is enhanced. The experimental technique and some theories at various levels of sophistication have been known for half a century [4, 5]. The main motivation was the development of polarized targets for particle research [6]. A typical target sample consists of small glassy frozen spheres of a suitable solvent (that provides the nuclei, usually protons or deuterons), to which suitable radicals (that provide the electron spin system) have been added. As such, the practical knowledge of these systems has been restricted to a few high-energy facilities in the world. A completely new, and by now very active, field was opened by the discovery [7] that it is possible to transform a typical target sample into a liquid solution, while retaining essentially all of the nuclear polarization. The method is known as dissolution-DNP. Molecules of interest, added in the solvent together with the radicals, see their nuclear polarization enhanced as well: and the product of dissolution-DNP is transferred to an NMR or MRI machine, where they can be studied with a much enhanced sensitivity (for a limited time: in the end the nuclear spin-lattice relaxation will drive the polarization back to its Boltzmann equilibrium value). It is therefore of interest to improve the theoretical understanding of this, in principle rather complex, process of DNP by thermal mixing. The hope would be to find guidelines for the choice of the optimal type and concentration of the radicals, so as to maximize the enhancement that can be obtained through dissolution-DNP. Apart from the proprietary tri-aryl-methyl (TAM) radicals used in the original work [7], quite some research, in targets as well as in dissolution-DNP, has been based on the widely available TEMPO (2,2,6,6-tetramethylpiperidine-1-oxyl) radical. The peculiarity of the latter is that it has quite some g-anisotropy, so that its ESR glassspectrum is rather wide. It was realized already in the context of target-research that this feature might hold a clue to some far-reaching implications in the theoretical treatment [5]. In particular, it can be argued that the electron 'Zeeman' Hamiltonian should refer only to the average ESR frequency, while the width of the spectrum is described by an 'offset' Hamiltonian. Again we have two commuting Hamiltonians that have (in the laboratory frame) no commensurate quanta, whereas in a Redfieldstyle rotating frame treatment they do.

It is the purpose of the present paper to show that a very similar approach, but allowing for partial saturation, can go a step further to quantitative agreement between experimental and calculated microwave spectra (a microwave spectrum displays the nuclear polarization, in our case of the $1-{ }^{13} \mathrm{C}$ in enriched acetate, as a function of the microwave frequency). We make an important extension, based on Provotorov's ideas [4], of the discussion in [1] concerning the model in [5]. We adhere more strictly to the rotating-frame concept [3], and allow for partial saturation. The available space for the present paper does not allow us to show that indeed the high-temperature limit of our model agrees (apart from some formal differences) with the steady-state solution of the well-known Provotorov equations. Our model takes the following as input parameters: 
Fig. 1 Calculated ESR lineshape of TEMPO radical in a field of $3.35 \mathrm{~T}$

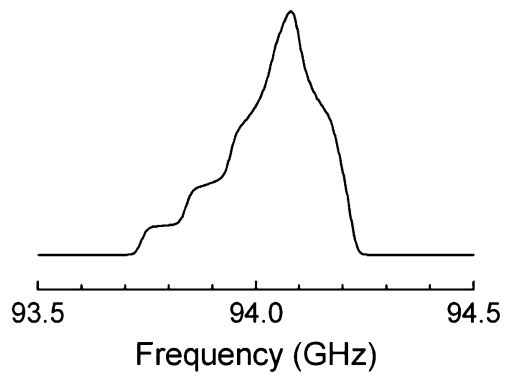

1. The ESR line-shape $f(\omega)$ (see Fig. 1).

2. The lattice temperature $T_{\mathrm{L}}$.

3. The microwave frequency $\omega_{\mathrm{m}}$.

4. The leakage parameter $f^{\mathrm{L}}$ (see Eq. 16 for definition).

5. The saturation rate $r_{\mathrm{s}}$ (see Eq. 20 for definition).

The items one to three are experimentally determined; the items four and five are treated as parameters, to be adjusted to obtain the best agreement between calculation and experiment. For convenience of discussion, we start by summarizing the ideas behind the Redfield thermodynamic treatment of the rotating-frame transformation. The expectation value $\langle A(t)\rangle$ of an observable described by an operator $A$ pertaining to a system described by a density operator $\rho(t)$ is given by

$$
\langle A(t)\rangle=\operatorname{Tr}(\rho(t) A)
$$

If the system has a (possibly time-dependent) Hamiltonian $H(t)$, the time evolution of $\rho(t)$ is given by the Liouville-von Neumann equation

$$
\frac{d \rho(t)}{d t}=-i \hbar[\rho(t), H]
$$

A stationary state should by definition be independent of $t$, and therefore have a time-independent Hamiltonian, so that $\rho(t)$ in Eq. 2 can settle (after an unknown time) to an (unknown) time-independent form $\rho$. Now, even though the microwave Hamiltonian is relatively small on the energy scale, it is essential for the DNP process, so we follow Redfield's idea of a rotating-frame transformation [3], where $H(t)$ becomes $H^{*}$ (independent of time) and $\rho(t)$ becomes $\rho^{*}(t)$ according to

$$
H^{*}=U_{\omega_{\mathrm{m}} t} \mathrm{HU}_{\omega_{\mathrm{m}} t}^{-1}-\omega_{\mathrm{m}} S_{\mathrm{Z}}
$$

and

$$
\rho^{*}(t)=U_{\omega_{\mathrm{m}} t} \rho(t) U_{\omega_{\mathrm{m}} t}^{-1}
$$

With $U_{\omega_{\mathrm{m}} t}=\exp \left(i \omega_{\mathrm{m}} t S_{\mathrm{z}}\right)$, so that

$$
\frac{d \rho^{*}}{d t}=-i \hbar\left[\rho^{*}, H^{*}\right]
$$

and the Redfield hypothesis is that in the stationary state, where the commutator in Eq. 5 is zero, $\rho^{*}$ will have some time-independent canonical form. To establish that 
canonical form, we follow to some extent ideas established in chapter 6F of [1], but stay much closer to Redfield thermodynamics. We follow Borghini's idea [3], namely (a) for a glass spectrum the energy in the rotating frame is mainly determined by the Zeeman-offset Hamiltonian; (b) in the energy equations the electron dipole-dipole term as well as the microwave Hamiltonian can be neglected, and (c) in the energy equations the nuclear Zeeman Hamiltonian must be included along with the electron Zeeman-offset Hamiltonian. The two additional steps we propose here are, first, the introduction of a common inverse temperature $\beta_{\mathrm{m}}$ for the combined nuclear Zeeman and electron offset-Zeeman reservoirs, and second, the introduction of an inverse temperature $\alpha_{\mathrm{m}}$ associated with the part of the laboratory frame electron Zeeman energy that is canceled through the transformation described in Eq. 4. The index $\mathrm{m}$ of $\alpha_{\mathrm{m}}$ and $\beta_{\mathrm{m}}$ indicates that their values depend on the microwave frequency $\omega_{\mathrm{m}}$. The physical reason justifying the hypothesis of a single common temperature $\beta_{\mathrm{m}}$ is that in a glassy matrix the spectral diffusion and the thermal mixing process equilibrating the nuclear Zeeman reservoir and the electron offset-Zeeman reservoir are very fast compared with all other relaxation mechanisms involved in the DNP process. As will be shown in the following, the original Borghini result is obtained by setting $\alpha_{\mathrm{m}}=0$, which corresponds to the case of large saturation rate $\left(r_{\mathrm{s}}=\infty\right.$, see below). We will assume that a stationary state is reached after a finite and reasonably short amount of time, on the order of hours. If we further assume that all five 'input parameters' of the model previously described are known, we are left with the task of solving for $\alpha_{\mathrm{m}}$ and $\beta_{\mathrm{m}}$. From $\beta_{\mathrm{m}}$, we will find the nuclear polarization according to the original Borghini reasoning and deduce the microwave spectrum. To accomplish this task we will write two energy balance equations under stationary conditions: in the laboratory frame and in the rotating frame. More precisely they are power equations, stating that the power going out is equal to the power coming in. In the lab frame, there is the saturating power of the microwave field, as in Provotorov theory [4]. In the rotating frame the microwave field is just a transverse component of the Zeeman field, and neglected with respect to the offset field. The electron Zeeman reservoir is heated by the Redfield mechanism and cooled through spin-lattice relaxation. Under stationary conditions, its temperature is somewhat above that of the lattice. The combined electron Zeemanoffset and nuclear Zeeman energy reservoir is on the one hand cooled by the Redfield mechanism, and on the other hand heated by the nuclear spin-lattice relaxation. Following [8] we will call the latter process "leakage" (but its physics is different from what was called by that name in the literature on DNP in inorganic crystals [2]; it is strictly defined by Eq. 16 below). The experimental goal is of course that the combined energy flows must lead to a cooling of the latter reservoir (note that spin systems can have positive and negative temperatures; the terms 'cooling' and 'heating' refer to the absolute value). The Zeeman Hamiltonian $H$ for one electron spin $S$ together with $N_{\mathrm{I}} / N_{\mathrm{S}}$ nuclear spins $I$ is

$$
H=\hbar \omega S_{\mathrm{z}}+\hbar \omega_{\mathrm{I}} \sum_{j=1}^{N_{\mathrm{S}} / N_{\mathrm{I}}} I_{\mathrm{z}, j}+H_{\mu \mathrm{w}}(t)
$$

With the rotating-frame transformation of Eq. 3 this becomes 


$$
\begin{aligned}
H^{*} & =\hbar\left(\omega-\omega_{\mathrm{m}}\right) S_{\mathrm{z}}+\hbar \omega_{\mathrm{I}} \sum_{j=1}^{N_{\mathrm{S}} / N_{\mathrm{I}}} I_{\mathrm{z}, j}+\hbar \omega_{1} S_{\mathrm{x}} \\
& \approx \hbar \Delta S_{\mathrm{z}}+\hbar \omega_{\mathrm{I}} \sum_{j=1}^{N_{\mathrm{S}} / N_{\mathrm{I}}} I_{\mathrm{z}, j}
\end{aligned}
$$

If all dipolar couplings are neglected, then the density matrix for this spin system is of the following form:

$$
\rho=\rho_{\mathrm{S}, \Delta} \prod_{j} \rho_{\mathrm{I}, j}
$$

where the tensorial product $\Pi$ has $N_{\mathrm{I}} / N_{\mathrm{S}}$ factors and $\rho_{\mathrm{S}, \Delta \Delta}$ and $\rho_{\mathrm{I}, \mathrm{j}}$ are the density matrices associated with an electron spin $S$ belonging to a homogeneous ESR line fitting at a frequency offset $\Delta$ from the microwave frequency $\omega_{\mathrm{m}}$, and its $N_{\mathrm{I}} / N_{\mathrm{S}}$ surrounding nuclear spins $I_{\mathrm{j}}$. The Ansatz is that in the rotating frame, a stationary state is attained after a generally unknown time, and that the forms of the two density matrices $\rho_{\mathrm{S}, \Delta}$ and $\rho_{\mathrm{I}, \mathrm{j}}$ are

$$
\begin{gathered}
\rho_{\mathrm{S}, \Delta}^{*} \propto \exp \left(-\alpha_{\mathrm{m}} \omega_{\mathrm{m}} S_{\mathrm{z}}-\beta_{\mathrm{m}} \Delta S_{\mathrm{z}}\right) \\
\rho_{\mathrm{I}, j}^{*} \propto \exp \left(-\beta_{\mathrm{m}} \Delta I_{\mathrm{z}}\right)
\end{gathered}
$$

and trivially, from the general relation expressed in Eq. 4, the laboratory frame density matrices $\rho_{\mathrm{S}, \Delta}=\rho_{\mathrm{S}, \Delta}^{*}$ and $\rho_{\mathrm{I}, j}=\rho_{\mathrm{I}, j}^{*}$. Since the relevant parts of the Hamiltonian are linear in $S_{Z}$ and $I_{Z}$ and the density matrices factorize, the total energy of the spin system is the sum of the individual energies so that, in the rotating frame,

$$
\begin{aligned}
E_{\Delta}^{*} & =-\frac{\hbar}{2} \Delta P_{\mathrm{S}}(\Delta)-\frac{\hbar}{2} \frac{N_{I}}{N_{\mathrm{S}}} \omega_{\mathrm{I}} P_{\mathrm{I}} \\
& =E_{\mathrm{S}, \Delta}^{*}+\frac{N_{I}}{N_{\mathrm{S}}} E_{\mathrm{I}}
\end{aligned}
$$

with

$$
\begin{gathered}
P_{\mathrm{S}}(\Delta)=\tanh \left(\frac{1}{2}\left(\alpha_{\mathrm{m}} \omega_{\mathrm{m}}+\beta_{\mathrm{m}} \Delta\right)\right) \\
P_{\mathrm{I}}=\tanh \left(\frac{1}{2} \beta_{\mathrm{m}} \omega_{\mathrm{I}}\right)
\end{gathered}
$$

We now extend this equation to an ensemble of $N_{\mathrm{S}}$ electron spins, for a normalized ESR line-shape function $f(\Delta)$ and $N_{\mathrm{I}}$ nuclear spins. Under the assumption of fast electron spin spectral diffusion compared with the electron spin lattice relaxation, a unique spin temperature is established for all electron spins. So $\beta_{\mathrm{m}}$ is constant across the inhomogeneous ESR line. In the stationary state, the energy is constant; therefore, 


$$
\int_{-\infty}^{\infty} f(\Delta)\left(\Delta \frac{P_{\mathrm{S}}(\Delta)-P_{\mathrm{S}, 0}(\Delta)}{T_{1, \mathrm{~S}}}+\frac{N_{\mathrm{I}}}{N_{\mathrm{S}}} \omega_{I} \frac{P_{\mathrm{I}}-P_{\mathrm{I}, 0}}{T_{1, \mathrm{I}}(\Delta)}\right) d \Delta=0
$$

If we call $T_{1, \mathrm{I}, 0}$ the nuclear spin relaxation time at low ('zero') electron spin polarization, then we have $[1,8]$

$$
\frac{1}{T_{1, \mathrm{I}}(\Delta)}=\frac{1}{T_{1, \mathrm{I}, 0}}\left(1-P_{\mathrm{S}}^{2}(\Delta)\right)
$$

We define a 'leakage' factor

$$
f^{\mathrm{L}}=\frac{N_{\mathrm{I}}}{N_{\mathrm{S}}} \frac{T_{1, \mathrm{~S}}}{T_{1, \mathrm{I}, 0}}
$$

and Eq. 14 becomes

$$
\int_{-\infty}^{\infty} f(\Delta)\left(\Delta\left(P_{\mathrm{S}}(\Delta)-P_{\mathrm{S}, 0}(\Delta)\right)+f^{\mathrm{L}} \omega_{\mathrm{I}}\left(P_{\mathrm{I}}-P_{\mathrm{I}, 0}\right)\left(1-P_{\mathrm{S}}^{2}(\Delta)\right)\right) d \Delta=0
$$

This equation contains the two temperature variables $\alpha_{\mathrm{m}}$ and $\beta_{\mathrm{m}}$. One needs another equation to solve the system. In the laboratory frame, the total electron spin Zeeman energy is equal to

$$
E_{\mathrm{lab}}=-\frac{\hbar}{2} \int_{-\infty}^{\infty} f(\Delta)\left(\omega_{\mathrm{m}}+\Delta\right) P_{\mathrm{S}}(\Delta) d \Delta
$$

in the stationary state, $\rho$ and $\rho^{*}$ are the same. If $W=\frac{\pi}{2} \omega_{1}^{2} f(0)$ (where $f(0)$ is the normalized amplitude at the microwave frequency) is the transition rate driven by the microwaves, the variation of the electron spin Zeeman energy is canceled in the stationary state:

$$
0=-\frac{\hbar}{2} \int_{-\infty}^{\infty} f(\Delta)\left(\omega_{\mathrm{m}}+\Delta\right) \frac{P_{\mathrm{S}}(\Delta)-P_{\mathrm{S}, 0}(\Delta)}{T_{1, \mathrm{~S}}} d \Delta-\hbar W \omega_{\mathrm{m}} P_{\mathrm{S}}(0)
$$

where $P_{\mathrm{S}}(0)=\tanh \left(\alpha_{\mathrm{m}} \omega_{\mathrm{m}} / 2\right)$ is the polarization at the microwave frequency. Introducing a parameter $r_{\mathrm{s}}$ that we call the 'saturation rate'

$$
r_{\mathrm{S}}=2 \pi \omega_{1}^{2} T_{1, \mathrm{~S}}
$$

we have

$$
\int_{-\infty}^{\infty} f(\Delta)\left(\omega_{\mathrm{m}}+\Delta\right)\left(P_{\mathrm{S}}(\Delta)-P_{\mathrm{S}, 0}(\Delta)\right) d \Delta=-r_{\mathrm{s}} f(0) \omega_{\mathrm{m}} P_{\mathrm{S}}(0)
$$

The set of the two Eqs. 17 and 21 can be numerically solved to extract $\alpha_{\mathrm{m}}$ and $\beta_{\mathrm{m}}$, with the leakage $f^{L}$ and the saturation rate $r_{\mathrm{s}}$ as parameters. In Fig. 2 we show three curves at each of our two experimental magnetic fields. The continuous lines 
Fig. 2 DNP microwave spectra measured in a field of 3.35 and $5.0 \mathrm{~T}$. For the parameters used to calculate theoretical curves, see the text
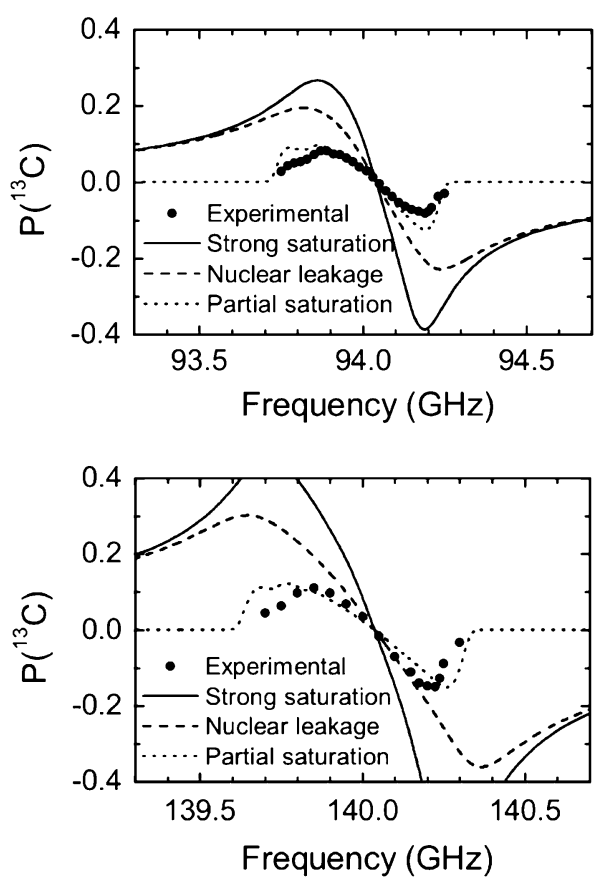

represent the original Borghini model: $f^{L}=0$ and $r_{\mathrm{s}} \rightarrow \infty[8,9]$. This model already captures an interesting feature of the experimental data: the negative polarization is larger than the positive one (in particular visible at $5 \mathrm{~T}$ ), but it lacks in quantitative agreement. The long-dashed lines are the equivalent of a variant that de Boer [8] has applied (to another radical, and at much lower temperatures), with a finite leakage, but still with infinite saturation. Both our curves have been drawn for $f^{L}=0.5$ and $r_{\mathrm{s}} \rightarrow \infty$. The value 0.5 has been chosen rather arbitrarily, but is similar to the value 0.3 for which [8] gave some experimental evidence. As should be expected, the addition of the leakage diminishes the polarizations; but the model still has unrealistically large wings. Finally, the short-dashed lines add a finite value for the saturation rate: $f^{\mathrm{L}}=0.5$ and $r_{\mathrm{s}}=2 \pi \times 197 \mathrm{MHz}$ at $3.35 \mathrm{~T} ; f^{\mathrm{L}}=0.5$ and $r_{\mathrm{s}}=2 \pi \times 62 \mathrm{MHz}$ at $5 \mathrm{~T}$. This model clips the wings, and even represents to some extent the structure in the positive polarization at $3.35 \mathrm{~T}$ (which is due to hyperfine interactions in the ESR spectrum). It is important to note that the addition of a single "fit parameter" improves the fit at many experimental points. This is our main reason to believe that finite saturation is an essential feature of our experiments. At the much lower bath-temperature used in [8] this effect may have been much smaller (electron spin-lattice relaxation times go up very fast when the temperature is lowered).

Of course this brings up immediately the question as to what would have happened if we had increased our experimental microwave power and also whether any realistic a priori estimates could be made for the parameters $f^{L}$ and $r_{\mathrm{s}}$. To start with the latter part, the two "fit-parameters" depend on three unknown quantities: 
Fig. 3 A modeling of the effect of heating by increasing the microwave power and experimental data (filed circle) obtained at $5 \mathrm{~T}$. The main hypothesis is that the sample temperature goes up as in the dotted line in (b); there is some theoretical justification to think that the electron $T_{1}$ then will vary as in (d). The panel (c) is the simple parabolic dependence of microwave field on microwave power. The dotted lines in (b) to (d) then predict the dotted line in (a). For cautionary remarks, see the text
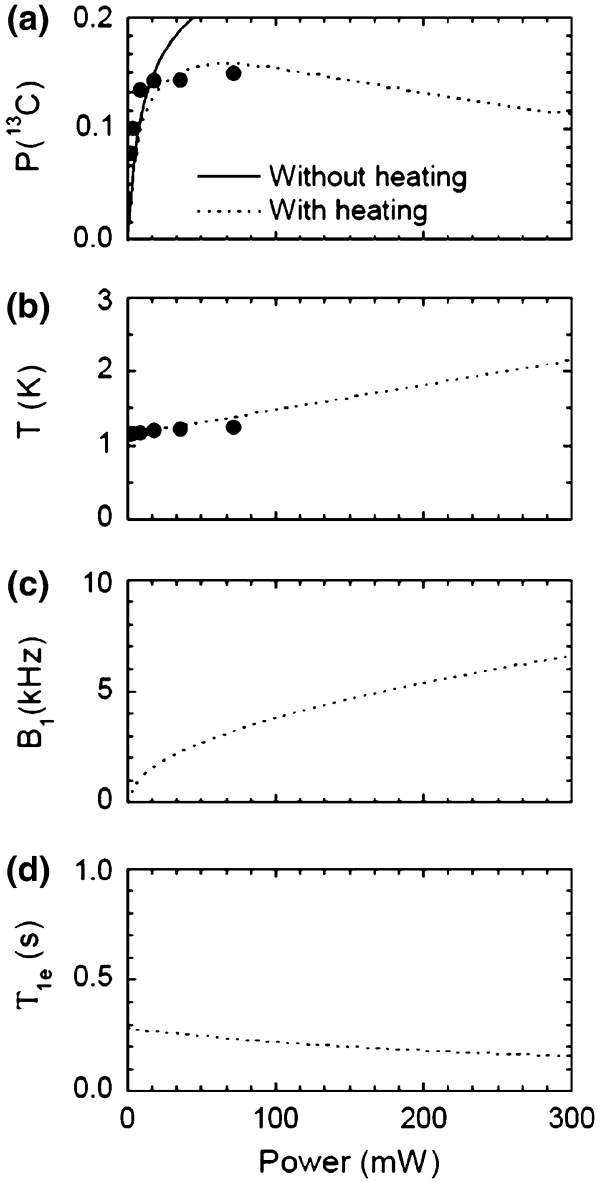

the strength of the microwave field $\omega_{1}$, and the electron and nuclear spin-lattice relaxation times. Concerning $\omega_{1}$, we can remark that DNP experiments are not done in a resonant cavity and that anyway we only know the microwave power at the entrance of a fairly long slightly oversized circular waveguide (data in Fig. 2 were obtained at $30 \mathrm{~mW}$ ). Concerning the relaxation times, such theories as exist refer to paramagnetically dilute systems, whereas any Borghini-based argument assumes couplings strong enough for thermodynamic considerations to hold. In a weak sense, $f^{L}$ and $r_{\mathrm{s}}$ are "thermodynamic" parameters that hide the many microscopic complexities of a system of coupled electron and nuclear spins. So if in the following we nevertheless propose values for those unknown experimental quantities, they should be considered as illustrative only.

Qualitatively, the importance of $f^{\mathrm{L}}$ can be shown from a comparison of data for samples with protonated and deuterated solvents [10]. There are reasons [1] to think that $f^{\mathrm{L}}$ varies with the square of the nuclear $\gamma$. In a qualitative comparison, one might say that with protonated solvents there will be "big" leakage, and with deuterated solvents "zero" leakage. Indeed [10] found that the ${ }^{13} \mathrm{C}$ polarization in the two types 
of solvents varies by a factor of two; again qualitatively, this is captured by the difference of the full curves and the long-dashed curves in Fig. 2, but note that our experiments were done in deuterated solvents, and in our case, the remaining protons are mainly on the acetate molecules.

Concerning the saturation rate, one might have hoped (see Eq. 20) that it simply goes up linear in the microwave power. However, as we have shown before [9], the bath temperature goes up with increasing microwave power; this will affect the spin-lattice relaxation times and the equilibrium electron spin polarization. So, with many precautions, we present in Fig. 3 a modeling of these temperature effects. The top panel gives the experimental extremal (the negative value in Fig. 2) ${ }^{13} \mathrm{C}$ polarization as function of microwave power at the input to the waveguide. The full curve is a calculation of what would be expected if only $r_{\mathrm{s}}$ would go up linear in the microwave power. The dotted curve in that panel gives the calculated results if the sample temperature goes up as in the dotted curve in the second panel (a kind of fit to the experimental points in that panel). The third panel gives our estimated values for $\omega_{1}$; and the fourth panel our estimated value for the electron spin-lattice relaxation time and its temperature-variation according to the second panel. Not shown is our estimated value for $T_{1, \mathrm{I}, 0}$ as per Eq. 16, but if we assume that the main leakage channel are the protons on the acetate molecules, then their spin-lattice relaxation comes out at several hundred seconds. It is just for the sake of argument that Fig. 3 shows calculated extrapolations to very high microwave powers; experimentally such sources are not readily available. But, acknowledging all doubts that went into the modeling of Fig, 3, it shows at least that the general suggestion of partial saturation (competing with sample heating) as the limit to the obtainable polarization in our systems can be described in a fairly consistent way.

We believe that our modeling in Fig. 2 shows once more the amazing power of thermodynamic reasoning [3-5] to describe the behavior of strongly interacting spin systems under external perturbations.

Acknowledgments The authors wish to acknowledge Dr. Ben van den Brandt, Dr. J. A. (Ton) Konter and Dr. Patrick Hautle of the Paul Scherrer Institute for the design and construction of the DNP cryostat. This work was supported by the Swiss National Science Foundation (Grants No. 200021-109479 and PP00P2_133562). Part of the experimental work was done while the authors were in the Laboratory of the Physics of Nanostructured Materials (LPMN) of the EPFL. Sami Jannin additionally acknowledges support from Prof. Geoffrey Bodenhausen, the Commission pour la Technologie et l'Innovation (CTI Grant 9991.1 PFIW-IW), and SNF (Grant No. 200020-124694).

\section{References}

1. A. Abragam, M. Goldman, Nuclear magnetism: order and disorder. Int. Ser. Monographs. Phys. (Clarendon Press, Oxford, 1982)

2. V.A. Atsarkin, Dynamic nuclear-polarization in dielectric solids. Uspekhi. Fizicheskikh. Nauk. 126(1), 3-39 (1978)

3. A.G. Redfield, Nuclear magnetic resonance saturation and rotary saturation in solids. Phys. Rev. 98(6), 1787-1809 (1955)

4. B.N. Provotorov, Magnetic resonance saturation in crystals. Sov. Phys. JETP 14(5), 1126-1131 (1962) 
5. M. Borghini, Spin-temperature model of nuclear dynamic polarization using free radicals. Phys. Rev. Lett. 20(9), 419 (1968)

6. S. Mango, Early target material research with chemical dopants. Nucl. Instrum. Meth. A 526(1-2), 1-6 (2004)

7. J.H. Ardenkjaer-Larsen, B. Fridlund, A. Gram, G. Hansson, L. Hansson, M.H. Lerche, R. Servin, M. Thaning, K. Golman, Increase in signal-to-noise ratio of $>10,000$ times in liquid-state NMR. P. Natl. Acad. Sci. USA 100(18), 10158-10163 (2003)

8. W.D. Boer, Dynamic orientation of nuclei at low-temperatures. J. Low Temp. Phys. 22(1-2), 185-212 (1976)

9. S. Jannin, A. Comment, F. Kurdzesau, J.A. Konter, P. Hautle, B. van den Brandt, J.J. van der Klink, A $140 \mathrm{GHz}$ prepolarizer for dissolution dynamic nuclear polarization. J. Chem. Phys. 128(24), 241102 (2008)

10. F. Kurdzesau, B. van den Brandt, A. Comment, P. Hautle, S. Jannin, J.J. van der Klink, J.A. Konter, Dynamic nuclear polarization of small labelled molecules in frozen water-alcohol solutions. J. Phy. D. Appl. Phy. 41(15), 155506 (2008) 